\title{
Parámetros de selección para el rendimiento en melón (Cucumis melo) cultivado bajo invernadero
}

\author{
José Eladio Monge Pérez ${ }^{1}$ \& Michelle Loría-Coto ${ }^{2}$ iD
}

1. Universidad de Costa Rica, Estación Experimental Agrícola Fabio Baudrit Moreno, Alajuela, Costa Rica; melonescr@yahoo.com.mx

2. Universidad Estatal a Distancia, Escuela de Ciencias Exactas y Naturales, Sabanilla, Costa Rica; michelle_loria@yahoo.com

Recibido 26-V-2020 • Corregido 05-IX-2020 • Aceptado 20-X-2020

DOI: https://doi.org/10.22458/urj.v12i2.2935

\begin{abstract}
Selection parameters for yield in muskmelon (Cucumis melo) under greenhouse conditions". Introduction: Muskmelon is among Costa Rica's main agricultural export products, and selection parameters for yield, considering direct and indirect effects, are best studied by path coefficient analysis, which divides correlation coefficients into direct and indirect effect components. Objective: To obtain a Path analysis for greenhouse Muskmelon yield. Methods: we planted the specimens on coconut fiber as substrate, managed with fertigation. The variables were: days to start of harvest (days after transplant-dat), fruit weight (FW, in $\mathrm{g}$ ), number of fruits per plant (NFP), yield ( $\mathrm{kg} / \mathrm{plant}$ ), fruit flesh firmness (N), percentage of total soluble solids ( ${ }^{\circ} \mathrm{Brix}$ ), equatorial fruit diameter (ED, in $\mathrm{mm}$ ), polar fruit diameter (PD, in $\mathrm{mm}$ ), $\mathrm{PD} / \mathrm{ED}$ ratio, fruit flesh thickness (FFT, in $\mathrm{mm}$ ), fruit cavity thickness (FCT, in $\mathrm{mm}$ ), and 2FFT/FCT ratio. Results: Yield showed a positive and highly significant correlation with NFP $\left(r=0,97^{* *}\right), P D / E D$ ratio $\left(r=0,65^{* *}\right)$ and PD $\left(r=0,54^{* *}\right)$. The main positive direct effects on yield were exhibited by NFP (path coefficient$P C=1,05), F W(P C=0,20), P D(P C=0,16), 2 F F T / F C T$ ratio $(P C=0,11)$ and $F C T(P C=0,06)$, while maximum positive indirect effects on yield were exhibited by $P D / E D$ ratio $(P C=0,69)$ and $P D(P C=0,48)$ through NFP. The main negative direct effects on yield were exhibited by $P D / E D$ ratio $(P C=-0,19)$, FFT $(P C=-0,15)$ and $E D(P C=-0,05)$. Conclusion: Under these conditions, number of fruits per plant was the main yield contributing variable.
\end{abstract}

Keywords: Cucumis melo, number of fruits per plant, yield, Pearson correlation, path analysis.
RESUMEN. Introducción: El melón se encuentra entre los principales productos agrícolas de exportación de Costa Rica, y los parámetros de selección de rendimiento, considerando los efectos directos e indirectos, se estudian mejor mediante el análisis de "coeficientes de sendero", que divide los coeficientes de correlación en componentes de efectos directos e indirectos. Objetivo: obtener un análisis de ruta para el rendimiento del melón en invernadero. Métodos: El cultivo se manejó con fertirrigación, en sustrato de fibra de coco. Las variables fueron: edad al inicio de cosecha (días después del trasplante-ddt), peso del fruto (PF, en $\mathrm{g}$ ), número de frutos por planta (NFP), rendimiento ( $\mathrm{kg} /$ planta), firmeza de pulpa del fruto $(\mathrm{N})$, porcentaje de sólidos solubles totales ( ${ }^{\circ}$ Brix), diámetro ecuatorial del fruto ( $D E$, en $\mathrm{mm}$ ), diámetro polar del fruto (DP, en $\mathrm{mm})$, relación $D P / D E$, grosor de pulpa del fruto (GP, en $\mathrm{mm}$ ), grosor de cavidad del fruto (GC, en $\mathrm{mm}$ ), y relación $2 \mathrm{GP} / \mathrm{GC}$. Resultados: Se obtuvo una correlación positiva y altamente significativa entre el rendimiento y: NFP $\left(r=0,97^{* *}\right)$, relación $D P / D E$ $\left(r=0,65^{* *}\right)$ y DP $\left(r=0,54^{* *}\right)$. El principal efecto directo positivo sobre el rendimiento fue ejercido por NFP (coeficiente de sendero-CS $=1,05)$, seguido por $P F$ $(\mathrm{CS}=0,20), \mathrm{DP}(\mathrm{CS}=0,16)$, relación $2 \mathrm{GP} / \mathrm{GC}(\mathrm{CS}=0,11)$, y $\mathrm{GC}$ $(C S=0,06)$, mientras que los máximos efectos indirectos positivos sobre el rendimiento fueron ejercidos por relación $D P / D E(C S=0,69)$ y $D P(C S=0,48)$ a través de NFP. Las siguientes variables ejercieron un efecto directo negativo sobre el rendimiento: relación DP/DE $(C S=-0,19)$, GP $(C S=-0,15)$, y DE $(C S=-0,05)$. Conclusión: En estas condiciones, la principal variable que contribuyó al rendimiento fue el número de frutos por planta.

Palabras clave: Cucumis melo, número de frutos por planta, rendimiento, correlación de Pearson, análisis de sendero. 
El melón es una hortaliza que se consume como postre, y que se cultiva en todo el mundo. Es muy apetecida debido a su sabor, dulzura, y efecto refrescante. Es una buena fuente de fibra dietética, vitaminas y minerales (Tomar, Kulkari, \& Kakade, 2008). Este cultivo representa uno de los principales productos agrícolas de exportación en Costa Rica; la cosecha se produce principalmente entre enero y abril (época seca), y el producto que no cumple con los requerimientos para exportación es destinado al mercado nacional (Monge-Pérez, 2014). En este país se producen los siguientes tipos de melón: Cantaloupe, Amarillo, Charentais, Harper, Piel de Sapo, Galia, Honey Dew, y Orange Flesh (Monge-Pérez, 2014).

El énfasis principal en fitomejoramiento de melón es el desarrollo de variedades de alto rendimiento y buena calidad del fruto. El rendimiento del melón es un rasgo cuantitativo complejo, pues es gobernado por un gran número de genes, y es afectado considerablemente por el ambiente (Reddy, Begum, Sunil, \& Reddy, 2017; Ibrahim \& Ramadan, 2013); por lo tanto, el fitomejoramiento para el rendimiento debe ser realizado mediante el enfoque de componentes de rendimiento. La influencia de cada característica sobre el rendimiento puede ser conocida a través de la correlación y los estudios de sendero (Reddy et al., 2017).

Los estudios de correlación permiten conocer la relación (naturaleza y grado de asociación) entre los caracteres asociados con el crecimiento, rendimiento y calidad del cultivo, y también desarrollar criterios de selección para el mejoramiento (Shivaprasad, Vasant, Halesh, Buvaneshwari, \& Vinay, 2017; Tomar et al., 2008; Mehta, Singh, \& Bhalala, 2009; Reddy et al., 2017). En melón, dichos estudios revelan que el rendimiento por planta se ha correlacionado positivamente con el número de frutos por planta y con el peso del fruto, por lo que son considerados como parámetros de selección para el rendimiento (Shivaprasad et al., 2017).

Sin embargo, la correlación por sí misma no revela el panorama completo de la dependencia de un componente sobre otro. Por lo tanto, el entendimiento de los efectos directos e indirectos de las principales variables que contribuyen con el rendimiento, es de suma importancia para la selección de genotipos de alto rendimiento (Choudhary, Fageria, \& Dhaka, 2004; Ibrahim \& Ramadan, 2013; Reddy et al., 2017). El análisis de coeficiente de sendero brinda un mejor índice de selección que solamente el coeficiente de correlación, por medio de la separación de los coeficientes de correlación del rendimiento y sus componentes, en efectos directos e indirectos (Tomar et al., 2008; Mehta et al., 2009).

Cada variable tiene dos vías de acción: la influencia directa sobre el rendimiento, y el efecto indirecto a través de los componentes, los cuales no son revelados en los estudios de correlación. El análisis de coeficiente de sendero mide el efecto directo e indirecto, y permite la separación de los coeficientes de correlación en componentes de efecto directo e indirecto (Reddy et al., 2017; Feyzian, Dehghani, Rezai, \& Jalali, 2009; Mehta et al., 2009; Ibrahim \& Ramadan, 2013).

Varios investigadores han estudiado el análisis de sendero en el cultivo de melón (Choudhary et al., 2004; Feyzian et al., 2009; Mehta et al., 2009; Reddy et al., 2017; Tomar et al., 2008; Ibrahim \& Ramadan, 2013), y también sobre correlaciones en este cultivo (Kultur, Harrison, \& Staub, 2001; Rad, Allahdoo, \& Fanaei, 2010; Shivaprasad et al., 2017; Monge-Pérez \& Loría-Coto, 2019).

El objetivo de esta investigación fue establecer las correlaciones de Pearson entre doce variables, y obtener el análisis de sendero para el rendimiento, en 10 genotipos de melón cultivados bajo ambiente protegido, en Alajuela, Costa Rica. 


\section{MATERIALES Y MÉTODOS}

Se sembraron 10 genotipos de melón (Cucumis melo L.) (Cuadro 1), en condiciones hidropónicas, en el invernadero de Hortalizas de la Estación Experimental Agrícola Fabio Baudrit Moreno (EEAFBM), la cual está localizada en Barrio San José de Alajuela, Costa Rica, a una altitud de $883 \mathrm{msnm}$. Se utilizaron genotipos de cinco diferentes tipos de melón (dos híbridos por tipo), los cuales se consideraron interesantes por sus características de calidad de fruta.

\section{CUADRO 1}

Genotipos utilizados

\begin{tabular}{ccc}
\hline Genotipo & Tipo de melón & Procedencia \\
\hline Acclaim & Cantaloupe & Estados Unidos \\
Brilliant & Amarillo & España \\
HSR-4310 & Galia, pulpa verde & Estados Unidos \\
HSR-4366 & Galia, pulpa anaranjada & Estados Unidos \\
JMX-1411 & Charentais & Holanda \\
JMX-915 & Harper & Holanda \\
JMX-801 & Harper & Holanda \\
JMX-207 & Amarillo & Holanda \\
JMX-1137 & Cantaloupe & Estados Unidos \\
Oui & Charentais & Estados Unidos \\
\hline
\end{tabular}

El cultivo se realizó en sacos plásticos rellenos con fibra de coco, de $1 \mathrm{~m}$ de largo, $20 \mathrm{~cm}$ de ancho y $15 \mathrm{~cm}$ de altura. La distancia de siembra fue de $25 \mathrm{~cm}$ entre plantas, y $1,54 \mathrm{~m}$ entre hileras, para una densidad de 2,60 plantas $/ \mathrm{m}^{2}$. Las plantas se sujetaron por medio de dos mallas plásticas, ubicadas una a cada lado de las hileras de plantas. Las plantas se manejaron a libre crecimiento (es decir, no se realizaron podas). Se contó con polinización entomófila en el invernadero, por medio de una colmena de Apis mellifera y otra de Nannotrigona sp.

El almácigo se sembró el 12 de julio de 2014, y el trasplante se realizó el 26 de julio de 2014. La cosecha inició el 4 de octubre de 2014 (70 días después de trasplante-ddt) para los genotipos más precoces. La evaluación de los frutos se llevó a cabo hasta el 26 de noviembre de 2014 (123 ddt).

Se implementó un sistema de manejo integrado de plagas, y se utilizó un programa de fertirrigación validado en la EEAFBM para la producción de melón en invernadero; la composición de la solución nutritiva se describe en el Cuadro 2. El fertirriego se suministró a cada hora, entre las 7:00a.m. y las 4:00p.m.

Se evaluaron las siguientes variables:

a) Número de frutos por planta (NFP): se obtuvo al registrar el número total de frutos producidos en cada parcela, y dividir ese dato entre el número de plantas por parcela.

b) Peso del fruto (PF, en g): se midió el peso de cada uno de los frutos producidos, y se obtuvo el promedio.

c) Rendimiento (kg/planta): se contabilizó el peso de los frutos producidos por parcela, y se dividió ese dato entre el número de plantas por parcela.

d) Porcentaje de sólidos solubles totales (SST, en ${ }^{\circ}$ Brix): se midió el porcentaje de sólidos solubles totales de todos los frutos, y se obtuvo el promedio. 
e) Firmeza de pulpa del fruto ( $F$, en $N)$ : se midió la firmeza de la pulpa de todos los frutos, y se calculó el promedio.

f) Diámetro ecuatorial del fruto ( $\mathrm{DE}$, en $\mathrm{mm}$ ): se midió el diámetro ecuatorial de cada fruto, y se obtuvo el promedio.

g) Diámetro polar del fruto (DP, en $\mathrm{mm}$ ): se evaluó el diámetro polar (distancia entre la cicatriz del pedúnculo y la cicatriz pistilar) de cada fruto, y se calculó el promedio.

h) Relación DP/DE: se obtuvo al dividir el diámetro ecuatorial entre el diámetro polar para cada uno de los frutos, y se obtuvo el promedio.

i) Grosor de pulpa del fruto (GP, en $\mathrm{mm}$ ): se midió el grosor de la pulpa de cada fruto, y se calculó el promedio.

j) Grosor de cavidad del fruto (GC, en $\mathrm{mm}$ ): se evaluó el grosor de la cavidad de cada fruto, y se obtuvo el promedio.

k) Relación 2GP/GC: se obtuvo al dividir el grosor de la pulpa (multiplicado por 2) entre el grosor de la cavidad, para cada uno de los frutos, y se calculó el promedio.

I) Edad al inicio de cosecha (EIC, en ddt): se registró la fecha de inicio de la cosecha para cada parcela, y se calculó el número de días transcurridos desde el trasplante.

\section{CUADRO 2}

Composición de la solución nutritiva utilizada en el cultivo de melón bajo invernadero, según las diferentes etapas fenológicas (datos en dosis por litro)

\begin{tabular}{lccc}
\hline \multicolumn{1}{c}{ Fuente } & Etapa 1 & Etapa 2 & Etapa 3 \\
\hline \multicolumn{1}{c}{ Solución A } & & & \\
Sulfato de magnesio (mg) & 620 & 720 & 780 \\
Fe-EDTA (mg) & 20 & 20 & 20 \\
Fosfato monopotásico (mg) & 290 & 290 & 290 \\
\multicolumn{1}{c}{ Solución B } & & & \\
Nitrato de calcio (mg) & 680 & 740 & 800 \\
Nitrato de potasio (mg) & 180 & 290 & 370 \\
Sulfato de cobre (mg) & 0,8 & 0,8 & 0,8 \\
Sulfato de zinc (mg) & 1,0 & 1,0 & 1,0 \\
Manvert-Mn (ml) & 9,0 & 9,0 & 9,0 \\
Ácido bórico (mg) & 4,0 & 4,0 & 4,0 \\
Molibdeno mix (ml) & 0,002 & 0,002 & 0,002 \\
\hline
\end{tabular}

Nota: Etapa 1: trasplante a inicio de floración; Etapa 2: inicio de floración a inicio de fructificación; Etapa 3: inicio de fructificación a final del cultivo.

El peso de los frutos se obtuvo con una balanza electrónica marca Ocony, modelo TH-I-EK, de 5000,0 $\pm 0,1 \mathrm{~g}$ de capacidad. El porcentaje de sólidos solubles totales se determinó con un refractómetro manual marca Atago, modelo $\mathrm{N}-1 \mathrm{a}$, con una escala de $0,0-32,0 \pm 0,2^{\circ}$ Brix. Para la evaluación de la firmeza de pulpa del fruto se utilizó un penetrómetro portátil marca Effegi, modelo FT-327, con una capacidad de 12,5 $\pm 0,1$ kilogramo-fuerza, y los datos se multiplicaron por un factor de conversión de 9,806 para obtener el dato en Newtons $(\mathrm{N})$; se utilizó el puntero cuya base mide $7,5 \mathrm{~mm}$ de ancho. Para la evaluación del diámetro ecuatorial y polar de los frutos, y del grosor de pulpa y de cavidad del fruto, se utilizó un calibrador electrónico marca Pittsburgh, con una capacidad de $154,5 \pm 0,1 \mathrm{~mm}$.

Se utilizó un diseño experimental irrestricto al azar. La parcela consistió de 12 plantas (3 sacos), y la parcela útil consistió en las 8 plantas centrales de la parcela ( 2 metros lineales centrales 
de la parcela). Se establecieron cuatro repeticiones por tratamiento. Todos los frutos producidos dentro de la parcela útil fueron evaluados. Se calculó el coeficiente de correlación de Pearson ( $r$ ) entre las doce variables evaluadas. Además, se realizó el análisis de sendero para el rendimiento (variable dependiente), en cuyo caso se obtuvo el coeficiente de sendero (CS), tanto para los efectos directos como indirectos de cada variable.

\section{RESULTADOS}

En el Cuadro 3 se muestra el resumen estadístico de los valores obtenidos para cada una de las variables estudiadas. Se destaca el alto coeficiente de variación obtenido por NFP (CV=72,98\%) y por el rendimiento $(\mathrm{CV}=70,90 \%)$. Las otras variables que también mostraron una variabilidad importante de los datos fueron $\mathrm{F}(\mathrm{CV}=29,92 \%)$ y $\mathrm{PF}(\mathrm{CV}=23,02 \%)$.

En el Cuadro 4 se presentan los coeficientes de correlación de Pearson ( $r$ ), entre las variables evaluadas. De las 66 correlaciones de Pearson calculadas, 32 de ellas fueron no significativas, 8 fueron significativas $(p \leq 0,05)$, y 26 fueron altamente significativas $(p \leq 0,01)$. Por su magnitud, sobresalen las correlaciones obtenidas entre el rendimiento y NFP $\left(r=0,97^{* *}\right)$; entre DE y GP $\left(r=0,91^{* *}\right)$; entre DP y relación DP/DE $\left(r=0,85^{* *}\right)$; entre PF y DE $\left(r=0,84^{* *}\right)$; entre PF y GP $\left(r=0,82^{* *}\right)$; entre GP y relación GP/GC ( $\left.r=0,74^{* *}\right)$; entre PF y GC ( $\left.r=0,72^{* *}\right)$; y entre DE y GC $\left(r=0,70^{* *}\right)$.

\section{CUADRO 3}

Resumen estadístico de los valores obtenidos para cada variable en melón

\begin{tabular}{lcccccc}
\hline \multicolumn{1}{c}{ Variable } & Promedio & $\begin{array}{c}\text { Valor } \\
\text { mínimo }\end{array}$ & $\begin{array}{c}\text { Valor } \\
\text { máximo }\end{array}$ & Mediana & $\begin{array}{c}\text { Desviación } \\
\text { estándar }\end{array}$ & $\begin{array}{c}\text { Coeficiente de } \\
\text { variación (\%) }\end{array}$ \\
\hline PF (g) & 714,90 & 309,67 & 1064,50 & 683,45 & 164,60 & 23,02 \\
SST ( ${ }^{\circ}$ Brix) & 11,24 & 9,04 & 13,33 & 11,39 & 0,94 & 8,32 \\
NFP & 1,30 & 0,13 & 3,13 & 0,94 & 0,95 & 72,98 \\
Rendimiento (kg/planta) & 0,88 & 0,12 & 2,29 & 0,72 & 0,62 & 70,90 \\
F (N) & 25,40 & 14,61 & 52,95 & 23,24 & 7,60 & 29,92 \\
DE (mm) & 101,11 & 82,37 & 115,75 & 101,35 & 8,11 & 8,02 \\
DP (mm) & 111,83 & 82,43 & 157,13 & 109,59 & 14,33 & 12,81 \\
Relación DP/DE & 1,11 & 0,98 & 1,65 & 1,04 & 0,17 & 15,36 \\
GP (mm) & 33,53 & 23,53 & 41,58 & 33,17 & 4,16 & 12,41 \\
GC (mm) & 42,36 & 36,18 & 50,15 & 42,72 & 3,36 & 7,93 \\
Relación 2GP/GC & 1,60 & 1,25 & 1,91 & 1,61 & 0,16 & 9,74 \\
EIC (ddt) & 78,26 & 70 & 88 & 77 & 7,34 & 9,38 \\
\hline
\end{tabular}

En el Cuadro 5 se presentan los coeficientes de sendero (CS) estimados de los efectos directos e indirectos de las variables evaluadas sobre el rendimiento en melón, según el análisis de sendero. El principal efecto directo positivo fue ejercido por NFP $(C S=1,05)$. Además, las otras variables que ejercieron un efecto directo positivo relevante sobre el rendimiento fueron $\mathrm{PF}$ $(C S=0,20)$, DP $(C S=0,16)$, relación $2 \mathrm{GP} / \mathrm{GC}(\mathrm{CS}=0,11)$, y $\mathrm{GC}(\mathrm{CS}=0,06)$. Los principales efectos indirectos positivos sobre el rendimiento fueron ejercidos por relación $D P / D E(C S=0,69)$, y DP $(C S=0,48)$, a través de NFP. Esto indica que los frutos alargados (mayor DP y mayor relación DP/DE) producen más frutos por planta, y esto contribuye a un mayor rendimiento, por lo que son caracteres importantes para la selección genética en fitomejoramiento en melón. Otros efectos indirectos positivos sobre el rendimiento fueron ejercidos por GP $(C S=0,16)$, $D E(C S=0,16)$, y GC $(C S=0,14)$, a través de $P F$, y por $D P / D E(C S=0,13)$, a través de DP. 


\section{CUADRO 4}

Coeficientes de correlación de Pearson ( $r$ ) entre las variables en melón

\begin{tabular}{|c|c|c|c|c|c|c|c|c|c|c|c|}
\hline & SST & NFP & $\mathbf{R}$ & $F$ & $\mathrm{DE}$ & DP & DP/DE & GP & GC & GP/GC & EIC \\
\hline $\mathrm{PF}$ & $0,19^{\text {ns }}$ & $-0,34^{*}$ & $-0,16^{\mathrm{ns}}$ & $0,40^{* *}$ & $0,84^{* *}$ & $0,38^{*}$ & $-0,11^{\mathrm{ns}}$ & $0,82^{* *}$ & $0,72^{* *}$ & $0,43^{* *}$ & $0,09^{\text {ns }}$ \\
\hline SST & & $-0,19^{\text {ns }}$ & $-0,17^{\mathrm{ns}}$ & $0,14^{\text {ns }}$ & $0,30^{\text {ns }}$ & $-0,08^{\text {ns }}$ & $-0,22^{n s}$ & $0,27^{\text {ns }}$ & $0,22^{\text {ns }}$ & $0,14^{\text {ns }}$ & $0,09^{\text {ns }}$ \\
\hline NFP & & & $0,97^{* *}$ & $-0,56^{* *}$ & $-0,43^{* *}$ & $0,45^{* *}$ & $0,65^{* *}$ & $-0,41^{* *}$ & $-0,50^{* *}$ & $-0,08^{\text {ns }}$ & $-0,51^{* *}$ \\
\hline $\mathrm{R}$ & & & & $-0,51^{* *}$ & $-0,28^{\text {ns }}$ & $0,54^{* *}$ & $0,65^{* *}$ & $-0,26^{n s}$ & $-0,39^{*}$ & $0,03^{\text {ns }}$ & $-0,49^{* *}$ \\
\hline $\mathrm{F}$ & & & & & $0,32^{*}$ & $-0,02^{\mathrm{ns}}$ & $-0,20^{\mathrm{ns}}$ & $0,17^{\mathrm{ns}}$ & $0,43^{* *}$ & $-0,15^{\mathrm{ns}}$ & $-0,04^{\mathrm{ns}}$ \\
\hline $\mathrm{DE}$ & & & & & & $0,11^{\mathrm{ns}}$ & $-0,42^{* *}$ & $0,91^{* *}$ & $0,70^{* *}$ & $0,55^{* *}$ & $0,20^{\text {ns }}$ \\
\hline $\mathrm{DP}$ & & & & & & & $0,85^{* *}$ & $0,01^{\text {ns }}$ & $0,03^{\text {ns }}$ & $0,04^{\text {ns }}$ & $-0,27^{\mathrm{ns}}$ \\
\hline $\mathrm{DP} / \mathrm{DE}$ & & & & & & & & $-0,48^{* *}$ & $-0,35^{*}$ & $-0,26^{\mathrm{ns}}$ & $-0,37^{*}$ \\
\hline GP & & & & & & & & & $0,57^{* *}$ & $0,74^{* *}$ & $0,35^{*}$ \\
\hline GC & & & & & & & & & & $-0,12^{\text {ns }}$ & $-0,01^{\mathrm{ns}}$ \\
\hline $\mathrm{GP} / \mathrm{GC}$ & & & & & & & & & & & $0,38^{*}$ \\
\hline
\end{tabular}

Nota: PF: peso fruto (g); SST: porcentaje sólidos solubles totales ( ${ }^{\circ}$ Brix); NFP: número frutos por planta; R: rendimiento (kg/planta); F: firmeza pulpa (N); DE: diámetro ecuatorial (mm); DP: diámetro polar (mm); DP/DE: relación DP/DE; GP: grosor pulpa $(\mathrm{mm})$; GC: grosor cavidad $(\mathrm{mm})$; GP/GC: relación $2 \mathrm{GP} / \mathrm{GC}$; EIC: edad inicio cosecha (ddt); ns: no significativa; *: significativa $(p \leq 0,05) ; * *$ : altamente significativa $(p \leq 0,01)$.

\section{CUADRO 5}

Coeficientes de sendero (CS) estimados de los efectos directos (diagonal, en letra negrita) e indirectos de las variables sobre el rendimiento $(\mathrm{kg} /$ planta) en melón

\begin{tabular}{lcccccccccccc}
\hline & PF & SST & NFP & $\mathbf{F}$ & DE & DP & DP/DE & GP & GC & GP/GC & EIC & CR \\
\hline PF & $\mathbf{0 , 2 0}$ & $-0,002$ & $-0,36$ & 0,001 & $-0,04$ & 0,06 & 0,02 & $-0,12$ & 0,05 & 0,05 & 0,003 & $-0,16^{\text {ns }}$ \\
SST & $\mathbf{0 , 0 4}$ & $\mathbf{- 0 , 0 1}$ & $-0,20$ & 0,001 & $-0,02$ & $-0,01$ & 0,04 & $-0,04$ & 0,01 & 0,02 & 0,003 & $-0,17^{\text {ns }}$ \\
NFP & $-0,07$ & 0,002 & $\mathbf{1 , 0 5}$ & $-0,001$ & 0,02 & 0,07 & $-0,12$ & 0,06 & $-0,03$ & $-0,01$ & $-0,02$ & $0,97^{* *}$ \\
F & 0,08 & $-0,002$ & $-0,59$ & $\mathbf{0 , 0 0 1}$ & $-0,02$ & $-0,003$ & 0,04 & $-0,03$ & 0,03 & $-0,02$ & $-0,001$ & $-0,51^{* *}$ \\
DE & 0,16 & $-0,003$ & $-0,45$ & 0,001 & $-\mathbf{0 , 0 5}$ & 0,02 & 0,08 & $-0,14$ & 0,04 & 0,06 & 0,01 & $-0,28^{\text {ns }}$ \\
DP & 0,08 & 0,001 & 0,48 & $-0,001$ & $-0,01$ & $\mathbf{0 , 1 6}$ & $-0,16$ & $-0,002$ & 0,002 & 0,004 & $-0,01$ & $0,54^{* *}$ \\
DP/DE & $-0,02$ & 0,003 & 0,69 & $-0,001$ & 0,02 & 0,13 & $-\mathbf{0 , 1 9}$ & 0,07 & $-0,02$ & $-0,03$ & $-0,01$ & $0,65^{* *}$ \\
GP & 0,16 & $-0,003$ & $-0,44$ & 0,001 & $-0,05$ & 0,002 & 0,09 & $-\mathbf{0 , 1 5}$ & 0,04 & 0,08 & 0,01 & $-0,26^{\text {ns }}$ \\
GC & 0,14 & $-0,002$ & $-0,52$ & 0,001 & $-0,04$ & 0,005 & 0,06 & $-0,09$ & $\mathbf{0 , 0 6}$ & $-0,01$ & $-0,001$ & $-0,39^{*}$ \\
GP/GC & 0,08 & $-0,002$ & $-0,08$ & $-0,001$ & $-0,03$ & 0,01 & 0,05 & $-0,11$ & $-0,01$ & $\mathbf{0 , 1 1}$ & 0,01 & $0,03^{\text {ns }}$ \\
EIC & 0,02 & $-0,001$ & $-0,54$ & $-0,001$ & $-0,01$ & $-0,04$ & 0,07 & $-0,05$ & $-0,001$ & 0,04 & $\mathbf{0 , 0 3}$ & $-0,49^{* *}$ \\
\hline
\end{tabular}

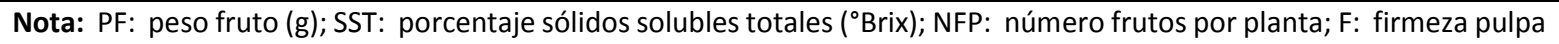
(N); DE: diámetro ecuatorial $(\mathrm{mm})$; DP: diámetro polar $(\mathrm{mm})$; DP/DE: relación DP/DE; GP: grosor pulpa $(\mathrm{mm})$; GC: grosor cavidad (mm); GP/GC: relación 2GP/GC; EIC: edad inicio cosecha (ddt); CR: correlación con el rendimiento; ns: no significativa; *: significativa $(p \leq 0,05) ; * *$ : altamente significativa $(p \leq 0,01)$.

Por otra parte, las siguientes variables ejercieron un efecto directo negativo sobre el rendimiento: relación $D P / D E(C S=-0,19), G P(C S=-0,15)$, y $D E(C S=-0,05)$. Además, los principales efectos indirectos negativos fueron ejercidos por $F(C S=-0,59)$, EIC $(C S=-0,54), G C(C S=-0,52), D E$ $(C S=-0,45), G P(C S=-0,44), P F(C S=-0,36)$, y SST $(C S=-0,20)$, en todos los casos a través de NFP. 


\section{CUADRO 6}

Coeficientes de sendero (CS) estimados de los efectos directos de las variables evaluadas, sobre el rendimiento ( $\mathrm{kg} /$ planta) en melón, informados en la literatura

\begin{tabular}{|c|c|c|}
\hline Variable & CS & Referencia \\
\hline \multirow{5}{*}{ EIC } & 0,045 & Feyzian et al., 2009 \\
\hline & 0,012 & Choudhary et al., 2004 \\
\hline & $-0,010$ & Reddy et al., 2017 \\
\hline & $-0,049$ & Mehta et al., 2009 \\
\hline & $-0,050$ & Tomar et al., 2008 \\
\hline \multirow{6}{*}{ PF } & 1,263 & Ibrahim \& Ramadan, 2013 \\
\hline & 0,927 & Feyzian et al., 2009 \\
\hline & 0,790 & Reddy et al., 2017 \\
\hline & 0,739 & Choudhary et al., 2004 \\
\hline & 0,150 & Tomar et al., 2008 \\
\hline & 0,149 & Mehta et al., 2009 \\
\hline \multirow{6}{*}{ NFP } & 0,902 & Mehta et al., 2009 \\
\hline & 0,900 & Tomar et al., 2008 \\
\hline & 0,759 & Feyzian et al., 2009 \\
\hline & 0,710 & Reddy et al., 2017 \\
\hline & 0,537 & Choudhary et al., 2004 \\
\hline & 0,198 & Ibrahim \& Ramadan, 2013 \\
\hline \multirow{3}{*}{ GC } & $-0,006$ & Feyzian et al., 2009 \\
\hline & $-0,010$ & Reddy et al., 2017 \\
\hline & $-0,017$ & Choudhary et al., 2004 \\
\hline \multirow{6}{*}{ GP } & 0,180 & Tomar et al., 2008 \\
\hline & 0,179 & Mehta et al., 2009 \\
\hline & 0,095 & Feyzian et al., 2009 \\
\hline & 0,010 & Reddy et al., 2017 \\
\hline & $-0,012$ & Choudhary et al., 2004 \\
\hline & $-0,143$ & Ibrahim \& Ramadan, 2013 \\
\hline \multirow{5}{*}{ SST } & 0,760 & Tomar et al., 2008 \\
\hline & 0,757 & Mehta et al., 2009 \\
\hline & 0,056 & Ibrahim \& Ramadan, 2013 \\
\hline & 0,001 & Reddy et al., 2017 \\
\hline & $-0,002$ & Choudhary et al., 2004 \\
\hline \multirow{5}{*}{ DP } & $-0,010$ & Reddy et al., 2017 \\
\hline & $-0,049$ & Ibrahim \& Ramadan, 2013 \\
\hline & $-0,070$ & Tomar et al., 2008 \\
\hline & $-0,073$ & Mehta et al., 2009 \\
\hline & $-0,388$ & Feyzian et al., 2009 \\
\hline \multirow{5}{*}{ DE } & 0,446 & Feyzian et al., 2009 \\
\hline & 0,290 & Tomar et al., 2008 \\
\hline & 0,286 & Mehta et al., 2009 \\
\hline & $-0,010$ & Reddy et al., 2017 \\
\hline & $-0,066$ & Ibrahim \& Ramadan, 2013 \\
\hline Relación DP/DE & 0,523 & Feyzian et al., 2009 \\
\hline
\end{tabular}


En el Cuadro 6 se presentan los CS obtenidos en el análisis de sendero por otros investigadores, para los efectos directos de las variables evaluadas, sobre el rendimiento en melón. Las variables PF, NFP y relación DP/DE mostraron CS positivos en todos los casos; DP obtuvo CS negativos en todos ellos; y las demás variables tuvieron CS positivos en unos casos, y CS negativos en otros. Todas esas investigaciones se llevaron a cabo a campo abierto.

En el Cuadro 7 se muestran las variables que ejercen los efectos directos (positivos y negativos) más importantes sobre el rendimiento en melón, según el análisis de sendero realizado en varias investigaciones.

\section{CUADRO 7}

Coeficientes de sendero (CS) estimados de los efectos directos (positivos y negativos) más importantes sobre el rendimiento ( $\mathrm{kg} /$ planta) en melón, según cada caso informado en la literatura

\begin{tabular}{|c|c|c|c|c|c|}
\hline \multirow{2}{*}{ Caso } & \multicolumn{2}{|c|}{ Efectos positivos (CS) } & \multicolumn{2}{|c|}{ Efectos negativos (CS) } & \multirow{2}{*}{ Referencia } \\
\hline & $1^{\circ}$ posición & $2^{\circ}$ posición & $1^{\circ}$ posición & $2^{\circ}$ posición & \\
\hline 1 & $\operatorname{PF}(0,739)$ & $\operatorname{NFP}(0,537)$ & $\mathrm{GC}(-0,017)$ & $\mathrm{GP}(-0,012)$ & Choudhary et al., 2004 \\
\hline 2 & $\operatorname{PF}(0,927)$ & $\operatorname{NFP}(0,759)$ & $\mathrm{DP}(-0,388)$ & GC $(-0,006)$ & Feyzian et al., 2009 \\
\hline 3 & $\operatorname{NFP}(0,900)$ & SST $(0,760)$ & $\mathrm{DP}(-0,070)$ & $\operatorname{EIC}(-0,050)$ & Tomar et al., 2008 \\
\hline 4 & $\operatorname{PF}(0,790)$ & $\operatorname{NFP}(0,710)$ & $\mathrm{GC}(-0,010)$ & DP $(-0,010)$ & Reddy et al., 2017 \\
\hline 5 & $\operatorname{PF}(1,263)$ & $\operatorname{NFP}(0,198)$ & $\mathrm{GP}(-0,143)$ & DE $(-0,066)$ & Ibrahim \& Ramadan, 2013 \\
\hline 6 & $\operatorname{NFP}(0,902)$ & SST $(0,757)$ & DP $(-0,073)$ & $\operatorname{EIC}(-0,049)$ & Mehta et al., 2009 \\
\hline
\end{tabular}

Nota: PF: peso fruto (g); SST: porcentaje sólidos solubles totales ( ${ }^{\circ}$ Brix); NFP: número frutos por planta; DE: diámetro ecuatorial $(\mathrm{mm})$; DP: diámetro polar $(\mathrm{mm})$; GP: grosor pulpa $(\mathrm{mm})$; GC: grosor cavidad $(\mathrm{mm})$; EIC: edad inicio cosecha (ddt).

\section{DISCUSIÓN}

La correlación hallada entre NFP y el rendimiento fue positiva y altamente significativa $\left(r=0,97^{* *}\right)$. En otro trabajo, se obtuvo un resultado similar para melones tipo Cantaloupe, Charentais, Galia y Harper $\left(r=0,84^{* *}-0,97^{* *}\right)$, pero dicha correlación fue no significativa para melones tipo Amarillo, Honey Dew y Japonés (Monge-Pérez \& Loría-Coto, 2019). De igual forma, otros investigadores encontraron que, entre ambas variables, la correlación fue positiva y significativa en algunos casos $\left(r=0,95^{*}-0,99 *\right)$, pero fue no significativa en otros, dependiendo del genotipo y del lugar del ensayo (Kultur et al., 2001). Otros investigadores también hallaron una correlación positiva y altamente significativa para ambas variables $\left(r=0,340 * *-0,703^{* *}\right)$ (Choudhary et al., 2004; Feyzian et al., 2009; Mehta et al., 2009; Reddy et al., 2017). Sin embargo, de forma contraria, otros autores obtuvieron una correlación negativa y altamente significativa $\left(r=-0,615^{* *}\right)$ (Ibrahim \& Ramadan, 2013). Y en otros estudios dicha correlación no fue significativa (Shivaprasad et al., 2017; Rad et al., 2010). Definitivamente, estos resultados sugieren que los genotipos involucrados en el análisis influyen en forma determinante en la correlación obtenida entre estas variables en cada estudio.

En la presente investigación se encontró una correlación positiva y altamente significativa entre DE y GP $\left(r=0,91^{* *}\right)$; otros investigadores hallaron resultados similares $\left(r=0,45^{* *}-0,87^{* *}\right)$ ( $\operatorname{Rad}$ et al., 2010; Reddy et al., 2017; Mehta et al., 2009; Feyzian et al., 2009). Sin embargo, otros autores obtuvieron una correlación negativa y altamente significativa entre dichas variables $(r=-0,449 * *)$ (Ibrahim \& Ramadan, 2013).

La correlación obtenida entre DP y relación DP/DE fue positiva y altamente significativa $\left(r=0,85^{* *}\right)$; en otro estudio se encontró un resultado similar $\left(r=0,72^{* *}\right)$ (Feyzian et al., 2009). 
En el presente trabajo se halló una correlación positiva y altamente significativa entre PF y $\mathrm{DE}\left(\mathrm{r}=0,84^{* *}\right)$; otros autores encontraron un resultado similar $\left(r=0,38^{* *}-0,87^{* *}\right)$ (Rad et al., 2010; Feyzian et al., 2009; Mehta et al., 2009; Reddy et al., 2017). Sin embargo, otros investigadores hallaron una correlación negativa y altamente significativa entre ambas variables $(r=-0,590 * *)$ (Ibrahim \& Ramadan, 2013). Esto significa que el genotipo de melón incluido en el análisis incide en forma importante en la correlación hallada entre estas dos variables, según la forma del fruto.

La correlación encontrada entre PF y GP fue positiva y altamente significativa $\left(r=0,82^{* *}\right)$; otros autores también hallaron una correlación positiva y significativa, o altamente significativa, entre dichas variables $\left(r=0,260 *-0,847^{* *}\right)$ (Rad et al., 2010; Ibrahim \& Ramadan, 2013; Mehta et al., 2009; Feyzian et al., 2009; Choudhary et al., 2004). Sin embargo, en otros estudios dicha correlación no fue significativa (Reddy et al., 2017; Shivaprasad et al., 2017), lo que parece indicar que un fruto que tenga una pulpa gruesa, no necesariamente tendrá un peso alto, sino que probablemente existen otras variables que inciden sobre el peso del fruto, como el diámetro ecuatorial y el diámetro polar del fruto.

En el presente ensayo se encontró una correlación significativa y negativa entre PF y NFP $\left(r=-0,34^{*}\right)$. En otro trabajo se halló un resultado similar para melón tipo Amarillo $\left(r=-0,87^{* *}\right)$, pero para otros seis tipos de melón la correlación entre estas variables no fue significativa (Monge-Pérez \& Loría-Coto, 2019). Otros investigadores también obtuvieron una correlación negativa y altamente significativa entre dichas variables ( $r$ entre $-0,590^{* *}$ y $-0,763^{* *}$ ) (Feyzian et al., 2009; Ibrahim \& Ramadan, 2013). Pero en otros estudios dicha correlación no fue significativa (Shivaprasad et al., 2017; Rad et al., 2010; Reddy et al., 2017; Mehta et al., 2009; Choudhary et al., 2004). Parece lógico que, entre mayor número de frutos logre cuajar una planta, menor será el peso da cada uno de esos frutos (relación inversamente proporcional), debido al mayor número de sumideros en que se deben repartir los fotoasimilados de la planta (Kultur, Harrison, \& Staub, 2001); sin embargo, no siempre la correlación entre dichas variables es significativa, y esto podría depender del genotipo, del manejo agronómico que se le brinde al cultivo, y de las condiciones climáticas.

La correlación obtenida entre PF y el rendimiento no fue significativa. En otro ensayo se halló un resultado similar en varios tipos de melón, excepto en el tipo Harper, en cuyo caso sí se halló una correlación positiva y significativa $\left(r=0,43^{*}\right)$ (Monge-Pérez \& Loría-Coto, 2019). Otros autores encontraron una correlación positiva y altamente significativa entre estas variables $\left(r=0,480^{* *}-0,986^{* *}\right)$ (Shivaprasad et al., 2017; Rad et al., 2010; Ibrahim \& Ramadan, 2013; Reddy et al., 2017; Mehta et al., 2009; Feyzian et al., 2009; Choudhary et al., 2004). Por lo tanto, en el resultado obtenido en esta correlación parece que existe una influencia determinante del genotipo de melón incluido en el análisis, así como de las condiciones ambientales y de manejo de las plantas.

Según varios investigadores, la asociación de caracteres revelada por el análisis de sendero puede estar influenciada por diferentes factores, como el germoplasma utilizado, el ambiente, y las variables usadas en el análisis (Feyzian et al., 2009; Reddy et al., 2017). Por lo tanto, la aplicabilidad general del análisis de sendero puede ser establecida por medio del análisis de datos de diferentes grupos de germoplasma bajo diferentes condiciones de producción (Reddy et al., 2017). Entonces, es posible argumentar que las diferencias entre los valores obtenidos en el presente ensayo y los datos informados en la literatura, se pueden deber a las diferencias en el material genético evaluado, en las condiciones ambientales (principalmente al ser un ensayo realizado bajo condiciones de invernadero, en contraposición a los otros que fueron llevados a cabo a campo abierto), así como en las variables seleccionadas para evaluación.

Al igual que en el presente trabajo, en otras dos investigaciones el principal efecto directo positivo se encontró para NFP (Tomar et al., 2008; Mehta et al., 2009). En otros cuatro trabajos, el principal efecto directo positivo fue ejercido por PF (Choudhary et al., 2004; Feyzian et al., 2009; 
Reddy et al., 2017; Ibrahim \& Ramadan, 2013); en el presente ensayo dicha variable ocupó la segunda posición, con un coeficiente relativamente bajo $(C S=0,20)$.

Con relación al principal efecto directo negativo sobre el rendimiento, al igual que en el presente ensayo (segunda posición), en otra investigación también dicho efecto fue hallado para GP (Ibrahim \& Ramadan, 2013).

En el presente ensayo, el efecto directo de NFP $(C S=1,05)$ fue casi igual a su coeficiente de correlación $(r=0,97)$ con el rendimiento; esta correlación explica la verdadera relación entre esa variable y el rendimiento, y puede indicar que la selección directa a través de este rasgo puede ser efectiva.

La presente investigación constituye el primer informe publicado sobre análisis de sendero en melón cultivado bajo invernadero. En estas condiciones, la actividad de los insectos polinizadores se puede ver afectada. Además, el tipo de expresión sexual del genotipo se convierte en una característica muy importante; las variedades andromonoicas producen más frutos por planta que las monoicas, en producción bajo invernadero (Monge-Pérez, 2016; Monge-Pérez \& Loría-Coto, 2019). Ambos aspectos influyen sobre la polinización y el cuaje de frutos, y pueden explicar la predominante influencia de NFP sobre el rendimiento en estas condiciones de cultivo.

La relación DP/DE mostró una correlación positiva altamente significativa con el rendimiento, pero su efecto directo fue negativo. En casos como este, los factores causales indirectos deben ser considerados simultáneamente para la selección (Reddy et al., 2017).

Los resultados obtenidos de la correlación y el análisis de sendero mostraron que la eficiencia en la selección para rendimiento en melón puede ser aumentada a través de la selección de NFP. Se concluye que NFP fue la variable principal que contribuyó con el rendimiento, debido a su efecto directo positivo y alto, y su correlación positiva y alta con el rendimiento por planta, y que es un criterio de selección fundamental en fitomejoramiento de melón cultivado bajo invernadero.

\section{AGRADECIMIENTOS}

Los autores agradecen la colaboración de Cristina Arguedas, Carolina Ramírez, Julio Vega y Andrés Oviedo en el trabajo de campo, y de Mario Monge en la revisión de la traducción del resumen al idioma inglés. Asimismo, agradecen el financiamiento recibido por parte de la Universidad de Costa Rica para la realización de este trabajo.

\section{ÉTICA, CONFLICTO DE INTERESES Y DECLARACIÓN DE FINANCIAMIENTO}

Los autores declaran haber cumplido con todos los requisitos éticos y legales pertinentes, tanto durante el estudio como en el manuscrito; que no hay conflictos de interés de ningún tipo, y que todas las fuentes financieras se detallan plena y claramente en la sección de agradecimientos. Asimismo, están de acuerdo con la versión editada final del documento. El respectivo documento legal firmado se encuentra en los archivos de la revista.

La contribución de los autores es como se detalla a continuación: J.E.M.P. y M.L.C. colaboraron en la misma proporción para la ejecución del trabajo de campo y la redacción del artículo. 


\section{REFERENCIAS}

Choudhary, B. R., Fageria, M. S., \& Dhaka, R. S. (2004). Correlation and path coefficient analysis in muskmelon (Cucumis melo L.). Indian Journal of Horticulture, 61(2), 158-162.

Feyzian, E., Dehghani, H., Rezai, A. M., \& Jalali, M. (2009). Correlation and sequential path model for some yield-related traits in melon (Cucumis melo L.). Journal of Agricultural Science and Technology, 11, 341-353.

Ibrahim, E. A., \& Ramadan, A. Y. (2013). Correlation and path coefficient analyses in sweet melon (Cucumis melo var. Aegyptiacus L.) under irrigated and drought conditions. Pakistan Journal of Biological Sciences, 16(13), 610-616.

Kultur, F., Harrison, H. C., \& Staub, J. E. (2001). Spacing and genotype affect fruit sugar concentration, yield, and fruit size of muskmelon. Hort Science, 36(2), 274-278.

Mehta, R., Singh, D., \& Bhalala, M. K. (2009). Correlation and path analysis in muskmelon. Indian Journal of Horticulture, 66(3), 396-399.

Monge-Pérez, J. E. (2014). Producción y exportación de melón (Cucumis melo) en Costa Rica. Tecnología en Marcha, 27(1), 93-103.

Monge-Pérez, J. E. (2016). Evaluación de 70 genotipos de melón (Cucumis melo L.) cultivados bajo invernadero en Costa Rica. InterSedes, 17(36), 1-41.

Monge-Pérez, J. E., \& Loría-Coto, M. (2019). Melón (Cucumis melo L.) cultivado bajo invernadero: correlaciones entre variables. Tecnología en Marcha, 32(1), 134-150.

Rad, M. R., Allahdoo, M., \& Fanaei, H. R. (2010). Study of some yield traits relationship in melon (Cucumis melo L.) germplasm gene bank of Iran by correlation and factor analysis. Trakia Journal of Sciences, 8(1), 27-32.

Reddy, B. P., Begum, H., Sunil, N., \& Reddy, M. T. (2017). Correlation and path coefficient analysis in muskmelon (Cucumis melo L.). International Journal of Current Microbiology and Applied Sciences, 6(6), 2261-2276.

Shivaprasad, M. K., Vasant, M. G., Halesh, G. K., Buvaneshwari, G., \& Vinay, G. M. (2017). Correlation studies in muskmelon for growth, yield and quality attributes. International Journal of Pure \& Applied Bioscience, 5(4), 1913-1916.

Tomar, R. S., Kulkarni, G. U., \& Kakade, D. K. (2008). Genetic analysis in muskmelon (Cucumis melo L.). Journal of Horticultural Sciences, 3(2), 112-118. 